\title{
BYPASS1: synthesis of the mobile root-derived signal requires active root growth and arrests early leaf development
}

\author{
Jaimie M Van Norman², Caroline Murphy ${ }^{1}$, Leslie E Sieburth ${ }^{1 *}$
}

\begin{abstract}
Background: The Arabidopsis bypass1 (bps1) mutant root produces a biologically active mobile compound that induces shoot growth arrest. However it is unknown whether the root retains the capacity to synthesize the mobile compound, or if only shoots of young seedlings are sensitive. It is also unknown how this compound induces arrest of shoot growth. This study investigated both of these questions using genetic, inhibitor, reporter gene, and morphological approaches.

Results: Production of the bps1 root-synthesized mobile compound was found to require active root growth. Inhibition of postembryonic root growth, by depleting glutathione either genetically or chemically, allowed seedlings to escape shoot arrest. However, the treatments were not completely effective, as the first leaf pair remained radialized, but elongated. This result indicated that the embryonic root transiently synthesized a small amount of the mobile substance. In addition, providing glutathione later in vegetative development caused shoot growth arrest to be reinstated, revealing that these late-arising roots were still capable of producing the mobile substance, and that the older vegetative leaves were still responsive.

To gain insight into how leaf development responds to the mobile signal, leaf development was followed morphologically and using the CYCB1,1::GUS marker for G2/M phase cells. We found that arrest of leaf growth is a fully penetrant phenotype, and a dramatic decrease in G2/M phase cells was coincident with arrest. Analyses of stress phenotypes found that late in development, bps1 cotyledons produced necrotic lesions, however neither hydrogen peroxide nor superoxide were abundant as leaves underwent arrest.

Conclusions: bps 1 roots appear to require active growth in order to produce the mobile bps 1 signal, but the potential for this compound's synthesis is present both early and late during vegetative development. This prolonged capacity to synthesize and respond to the mobile compound is consistent with a possible role for the mobile compound in linking shoot growth to subterranean conditions. The specific growth-related responses in the shoot indicated that the mobile substance prevents full activation of cell division in leaves, although whether cell division is a direct response remains to be determined.
\end{abstract}

\section{Background}

Plants synthesize a wide array of metabolites, and a major goal of metabolomics is to identify natural plant metabolites and their associated functions (reviewed in [1-3]). Recent advances facilitating identification of metabolites $[4,5]$ have led to identification of groups of metabolites that correlate with important plant traits,

\footnotetext{
* Correspondence: sieburth@biology.utah.edu

'Department of Biology, University of Utah, 257 South 1400 East, Salt Lake

City, Utah, 84112, USA

Full list of author information is available at the end of the article
}

such as growth rate and biomass [6,7], and identified metabolic regulators such as leucine [8]. However, how specific metabolites other than characterized hormones function in signaling and development is largely unknown. One approach to learning about alternate signaling molecules is to study mutants with signalingrelated defects.

The Arabidopsis bypass1 (bps1) mutant might be an important tool for identifying a metabolite functioning as a long-distance signal. The bps 1 mutant produces small abnormal roots and shoot development arrests

\section{Biomed Central}


soon after germination. This phenotype is linked to a mobile substance as the bps 1 mutant root is necessary to induce arrest of bps 1 shoots, and in graft chimeras, the bps 1 root is sufficient to induce arrest of the wildtype shoot [9]. These observations led to a model featuring BPS1 as a negative regulator that was required to prevent the excess production of a mobile substance. The mobile compound appears to be novel, and its synthesis requires carotenoid biosynthesis [10]. The pathway producing the bps 1 mobile compound appears to be conserved in plant lineages, as knock-downs of conserved BPS-like genes in tobacco produced similar phenotypes [11]. Critical questions include whether this mobile compound is an endogenous developmental regulator, and how it modifies shoot growth.

Control over shoot branching by a root-derived signal has been elegantly analyzed in pea, rice, and Arabidopsis [12-15]. In these systems, mutations disrupting biosynthetic enzymes lead to reduced production of a mobile compound that controls auxin transport in the shoot $[16,17]$. Recently, this substance was identified as strigolactone $[18,19]$. Additional unknown root-to-shoot signals have been implicated by studies of drought (reviewed in [20]), soil compaction [21], nutrient depletion [22-24] and low-fluence UV-B light [25]. The identities of the mobile compounds elicited by these treatments are unknown; it is also unknown whether the bps 1 mobile substance is related to any of these pathways, but its root-to-shoot mobility make it an attractive candidate.

It is also possible that the bps 1 mobile compound could instead be an intermediate molecule that normally doesn't accumulate. For example, a biosynthetic pathway might be blocked in bps 1 mutants, resulting in build-up of a precursor that happens to be mobile, and happens to have biological activity. For example, in superroot 1 mutants, a defect in glucosinolate biosynthesis causes a build-up of precursors that spills over into auxin biosynthesis, resulting in a high-auxin phenotype [26].

Here, we evaluate the conditions under which $b p s 1$ roots produce the mobile compound, and the characteristics of shoots undergoing arrest from this substance. We find that bps 1 roots produce and transport the mobile substance in actively growing roots, but that arrest of cell division leads to cessation of signaling to the shoot. Shoot responses include growth cessation, and in particular, arrest of cell division.

\section{Results}

The bps 1 root: shoot growth inhibition requires root growth

The central feature of bps 1 mutants is that a growtharresting mobile compound arises in the root [9]. However, the experimental basis for this assignment required wounding, and it was only tested in very young seedlings. To expand our understanding of the root's role in producing the bps 1 signal, we examined how leaf development responded when bps 1 root growth and development was blocked after embryogenesis. Post-embryonic root growth and development requires glutathione (GSH, [27]). Root development can therefore be blocked by either supplying germinating seeds with L-buthionine sulfoximine (BSO), an inhibitor of $\gamma$-glutamylcysteine synthetase, or by generating double mutants between bps 1 and root meristemless 1-1 (rml1-1), which has a defect in the gene encoding $\gamma$-glutamylcysteine synthetase and lacks post-embryonic root development $[27,28]$.

In agreement with previous publications, the roots of BSO-treated wild type appeared to arrest development at germination. In addition, these plants produced small, but flattened, leaves with distinct blade and petiole, a modest reduction in leaf vein pattern, and bleached cotyledons (Figure 1). By contrast, bps1 mutants grown

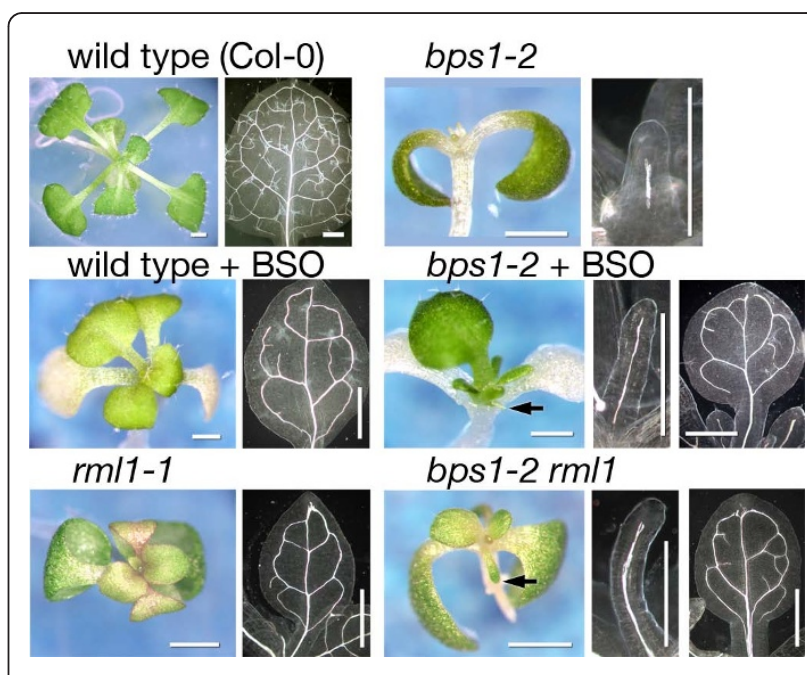

Figure 1 Sustained synthesis of the bps 1 mobile compound requires post-embryonic root growth. The top row shows wild type seedlings with their broad flat leaves and highly interconnected leaf veins, and bps $1-2$ mutants, with their very small radial leaf primordia and incomplete primary vein. The second row shows the effect of BSO-induced root arrest on wild type and bps 1 mutants. In the wild type, shoots of BSO-grown plants had chlorotic cotyledons and small leaves with modestly reduced vein patterns. The shoots of bps 1-2 mutants growth on BSO-containing media produced two types of leaves: the first leaf pair (black arrow) were elongated but radial, and contained only a single vein, while leaves that arose later were broad, flat, and produced more complex vein patterns. The third row shows $r m / 1-1$ and bps $1-2$ rm/1-1 double mutants. The rm/1-1 mutants were slightly smaller than the wild type and they produced slightly narrow, pointed leaves with modestly reduced patterns of veins. The bps 1-2 rm/1-1 double mutants produced two types of leaves: the first leaf pair (black arrow) were radialized but elongated, and contained a single vein, while later leaves were broad, flat, and produced complex vein patterns. Size bars: $500 \mu \mathrm{m}$. 
on BSO-supplemented medium produced leaves with two distinct shapes (Figure 1). The first leaf pair was small, radially symmetric, and contained only a single unbranched vein, while subsequently produced leaves were broad, flat, showed distinct blade and petiole, and contained both primary and secondary veins. This partial rescue of leaf development in BSO-treated bps 1 mutants suggested that post-germination arrest of the bps1 root led to reduced synthesis of the root mobile signal.

Similarly, the roots of rml1-1 mutants appeared to arrest development at germination, and they produced small, flattened leaves with distinct blade and petiole (Figure 1). Growth of rml1-1 mutants on GSHsupplemented medium restored post-embryonic root growth, as reported previously [27] (Table 1). The bps1 single mutants and F2 seedlings derived from $r m l 1-1 /+$ bps1-2/+ parents, grown on GSH-supplemented media, were indistinguishable from bps 1 controls (Table 1). By contrast, F2 seedlings derived from $r m l 1-1 /+$ bps1-2/+ parents grown on standard growth medium (lacking GSH), segregated for four different phenotypes: wild type; rml1-1; bps1-2; and a phenotype similar to BSOgrown bps1 mutants (Figure 1). This last phenotype appeared at numbers consistent with it being the rml1-1 bps1-2 double mutant (Table 1). As with rml1-1, the bps 1 rml1-1 double mutants produced roots that showed no sign of post-embryonic cell divisions. However, their first pair of leaves were radial and contained a single unbranched vein; these were similar to the bps1 single mutant, but much longer (Figure 1). Strikingly, leaf 3 and subsequently produced leaves were broad and flattened, with distinct petiole and blade, and contained both primary and secondary veins, much like the leaves of rml1-1 single mutants and BSO-treated bps 1 (Figure 1). Thus, these data indicate that post-embryonic root growth

Table 1 The bps 1 shoot phenotype requires post-embryonic root development

\begin{tabular}{|c|c|c|c|c|c|c|c|}
\hline \multirow[b]{2}{*}{$\begin{array}{c}\text { Plants } \\
\text { analyzed }\end{array}$} & \multirow[b]{2}{*}{ GSH } & \multirow[b]{2}{*}{$\begin{array}{c}\text { Total } \\
\text { (n) }\end{array}$} & \multicolumn{4}{|c|}{ Shoot Phenotypes Observed } & \multirow[b]{2}{*}{$\chi^{2}$} \\
\hline & & & $\begin{array}{c}\text { wild } \\
\text { type } \\
\text { (n) }\end{array}$ & $\begin{array}{c}r m / 1 \\
\text { (n) }\end{array}$ & $\begin{array}{l}\text { bps1 } \\
\text { (n) }\end{array}$ & $\begin{array}{c}\text { bps1 } \\
\text { rm/1 } \\
\text { (n) }\end{array}$ & \\
\hline$r m / 1-1$ & - & 240 & 189 & 51 & 0 & 0 & $1.8^{\mathrm{a}}$ \\
\hline rm/1-1 & + & 70 & 70 & 0 & 0 & 0 & $\mathrm{n} / \mathrm{a}$ \\
\hline bps1-2 & - & 224 & 164 & 0 & 60 & 0 & $0.381^{a}$ \\
\hline bps1-2 & + & 111 & 85 & 0 & 26 & 0 & $0.147^{a}$ \\
\hline $\begin{array}{c}r m / 1-1 \text { bps } 1-2 \\
\text { F2 }\end{array}$ & - & 1258 & 728 & 236 & 210 & 84 & $3.793^{\mathrm{b}}$ \\
\hline $\begin{array}{c}r m / 1-1 \text { bps } 1-2 \\
\text { F2 }\end{array}$ & + & 590 & 437 & 0 & 153 & 0 & $0.2734^{2}$ \\
\hline
\end{tabular}

$\mathrm{n} / \mathrm{a}$, not applicable.

${ }^{\text {a }}$ Critcal $\chi^{2}$ values at $95 \%$ confidence is 3.841 when $d f=1$.

${ }^{\mathrm{b}}$ Critical $\chi^{2}$ values at $95 \%$ confidence is 7.815 when $d f=3$. and development is required for continuous production and delivery of the leaf-arresting substance and further support the root as the source of this molecule [9].

Because imposing a GSH deficit (using either BSO or rml1-1) led to partial rescue of the bps1 shoot phenotype, it was a formal possibility that the bps1 rootderived compound could be GSH itself. To test this, we supplied GSH to excised bps1 shoots, and monitored subsequent leaf development. Typically, root excision leads to partially rescued shoot development in approximately $75 \%$ of bps 1 single mutants [9]. We reasoned that if the mobile signal was GSH, supplying it to bps 1 mutants following root excision would reduce the number of bps1 mutants that were rescued by root excision. However, supplying GSH did not diminish shoot developmental rescue, and no regimen of GSH provision (prior to cut, after cut, or both prior and after cut) yielded a statistically significant reduction of developmental rescue. Moreover, shoot rescue resulted in leaves reaching similar sizes, whether or not excised shoots were supplemented with GSH (Table 2). These data indicated that the mobile compound was not GSH.

\section{Arrested roots provide a transient source of the bps 1 signal}

Arrest of post-embryonic root growth in bps1 caused strikingly different responses in the first leaf pair as compared to leaf three. Both BSO-grown bps 1 mutants and rml1 bps 1 double mutants initially produced a pair of radialized leaves, yet rescued development was observed in subsequently produced leaves (Figure 1). In addition, the rml1-1 bps1-2 first leaf pair was consistently larger than that of BSO-grown bps1-2 mutants. These results contrast to root excision (carried out at day 4), where the strongest rescue was observed in the first leaf pair [9], and suggest that the arrested bps1 root might be a transient source of the bps 1 mobile compound.

We tested this possibility using the bps 1 temperature dependent phenotype [9]. We compared the first leaf pair of bps1 rml1-1 double mutants grown at 16, 22, and $29^{\circ} \mathrm{C}$. For bps 1 single mutants, leaf development is temperature dependent: severe arrest occurs at low temperatures, and small but flattened leaves are produced at high temperatures. Thus, if the radialized first leaf pair of bps 1 rml1-1 double mutants was due to the bps 1 mobile signal, then we expected its development to be similarly dependent on growth temperature.

Growth of bps $1 \mathrm{rml1}$ double mutants at $16^{\circ} \mathrm{C}$ led to production of a narrow radially-shaped first leaf pair that was much longer than the bps1-2 control (Figure 2). In bps $1 \mathrm{rml} 1$ double mutants grown at $22^{\circ} \mathrm{C}$, the first leaf pair was long, but very narrow. Its narrowness was 
Table 2 Analysis of GSH as a candidate for the BPS1-regulated signal

\begin{tabular}{|c|c|c|c|}
\hline \multicolumn{2}{|c|}{ Growth Medium } & \multirow[t]{2}{*}{ Total bps1 with excised root $(\mathrm{n})$} & \multirow[t]{2}{*}{ Percent producing broad leaves with distinct blade and petiole $(\mathrm{n})$} \\
\hline Pre-excision & Post-excision & & \\
\hline GSH- & GSH- & 31 & $77 \%(24)$ \\
\hline GSH- & $\mathrm{GSH}+$ & 40 & $83 \%(33)$ \\
\hline $\mathrm{GSH}+$ & GSH- & 27 & $70 \%(19)$ \\
\hline $\mathrm{GSH}+$ & GSH+ & 34 & $94 \%(32) * *$ \\
\hline
\end{tabular}

** The number of plants that produce leaves under GSH+/GSH+ conditions is statistically greater than the number that produces leaves under GSH-/GSHconditions ( $p$ value $=0.009$ ).

similar to the age-matched bps1 single mutant, but it was much longer. Finally, in bps 1 rml1 double mutants grown at $29^{\circ} \mathrm{C}$, the first leaf pair was flattened and had a distinct blade, very similar to the bps1 control. The similar effects of growth temperature on the first leaf pair of bps 1 single and bps $1 \mathrm{rml1}$ double mutants indicates that the shape of the first leaf pair is due to the bps1 mobile root-derived compound. This suggests that the first leaf pair of bps1 rml1 double mutants was exposed to the root-derived compound, while the later-arising rescued leaves were not.

\section{Competence to synthesize and respond to the bps 1 signal is retained in older seedlings}

Although the molecular target of the bps1 root-derived mobile compound is unknown, root-dependent arrest of early shoot development in bps1 seedlings indicates that the target is present at this early stage. However, we do not know if the molecular target is present later in development, nor do we know whether an older root retains the capacity to synthesize the mobile compound.

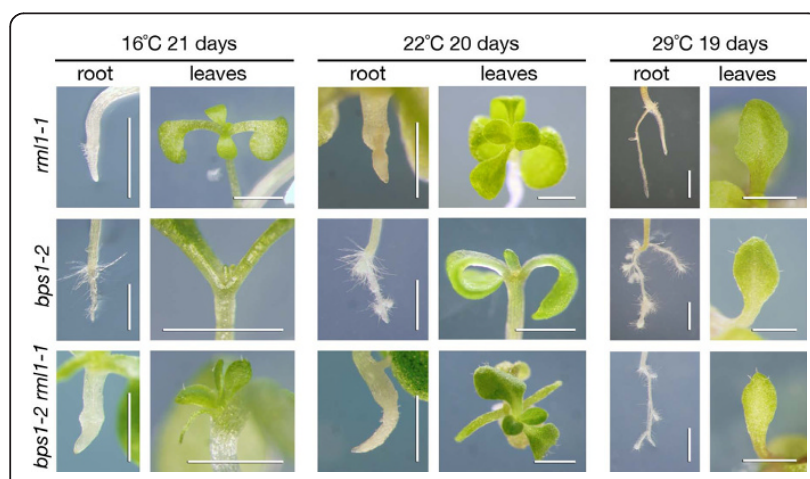

Figure 2 Temperature responsive phenotype of the bps $1 \mathrm{rm} / 1$ first leaf pair supports exposure to the bps 1 mobile compound during development. In bps 1 single mutants, leaf development arrests early at low temperature $\left(16^{\circ} \mathrm{C}\right)$, while growth at higher temperatures results in progressively more leaf growth. In bps $1-2$ rm/1-1 double mutants, development of the first leaf pair responds similarly to growth temperature. When grown at $16^{\circ} \mathrm{C}$, the first leaf pair was small and radial, growth at $22^{\circ} \mathrm{C}$ led to much longer, but still very narrow leaves, and growth at $29^{\circ} \mathrm{C}$ led to small but broad leaves. Note that the $r m / 1$ single mutant also showed enhanced root growth at the elevated temperature.
To test this, we followed up on the observation that rml1 root development is rescued by supplying glutathione (GSH) [27]. We reasoned that supplying GSH to an older bps $1 \mathrm{rml1}$ double mutant might restore growth of a bps1-like root. If this root retained the ability to synthesize and deliver the mobile compound, and its molecular target was present in older shoots, then we would expect to observe arrested leaf growth.

Seeds segregating for both bps 1 and $r m l 1-1$ were plated on standard growth media, and at 10 days, 90 seedlings with $r m l 1$ root phenotype were transferred to GSH-supplemented medium (approximately 22-23 were expected to be bps $1 \mathrm{rml1}$ double mutants). At 18 days after transfer (28 total days), we analyzed their phenotypes. Most of the plants looked the same as rml1-1 controls; they produced normal-appearing roots and large flat leaves (Figure 3). However, 18 of the seedlings

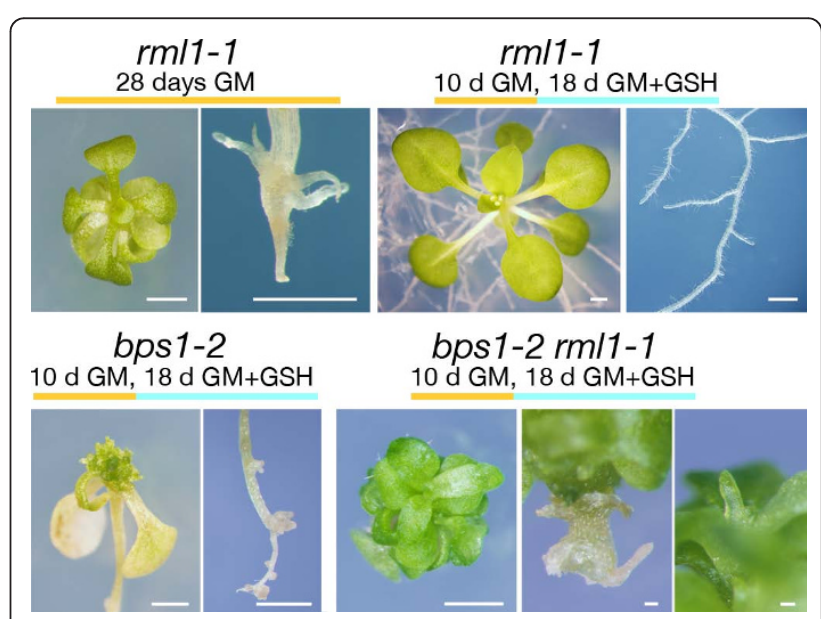

Figure 3 Restored root growth in bps1-2 rm/1-1 plants reinstates arrest of leaf development. Top row: $r m / 1-1$ grown for 28 days on normal growth medium (GM) (left) and or transferred to GSH-supplemented GM at day 10 (right). The small stunted root is rescued by transfer to GSH (+). Bottom row: bps 1-2 (left) and bps 1-2 rm/1-1 (right), both transferred from GM to GM+GHS at 10 days. The bps1 single mutants produced small roots and narrow radialized leaves. The bps $1-2 \mathrm{rm} / 1-1$ double mutants showed a novel phenotype. The roots enlarged radially, they produced a few enlarged lateral-root-like organs, and arrested leaf primordia accumulated at the shoot apex. Size bars $=1 \mathrm{~mm}$ except for bps $1-2$ rm/1-1 roots and leaf primordia, where bars $=0.1 \mathrm{~mm}$. 
produced roots that were short, blunt, and very swollen, and had produced lateral roots somewhat similar to bps1. These plants also produced shoots with variable leaf shapes. Their first leaf pair was long and radial, the subsequently produced 5-9 leaves appeared flat (partially rescued), and with distinct petiole and blades. Finally, the newest arising leaves were short and radially shaped (bps1-like). This range of leaf phenotypes is consistent with restored synthesis and delivery of the bps 1 mobile compound upon induction of root growth, and response in these later-arising vegetative leaves. These results indicate that roots retain the capacity to synthesize and deliver the bps1 mobile substance to the shoot, and that the shoots of older seedlings retain the ability to respond.

\section{The bps 1 mobile compound: synthesis and delivery} require neither the phloem nor endodermis

We next developed double mutants that combined bps1 with altered phloem development (apl), shortroot (shr), and scarecrow (scr) mutants. The apl mutant lacks phloem, shr lacks endodermis, and scr replaces endodermal and cortical cell layers with a single layer of mixed identity [29-33]. We predicted that if the phloem or endodermis were the sole site of synthesis of the bps 1 mobile compound, or required for its transmission, then leaf development would be at least partially restored in the double mutants.

The bps 1 apl double mutants showed an arrested shoot phenotype that was indistinguishable from bps 1 (Figure 4), indicating that phloem was dispensable for synthesis and delivery of the bps 1 mobile compound, at least in these very small mutants. Similarly, both shr

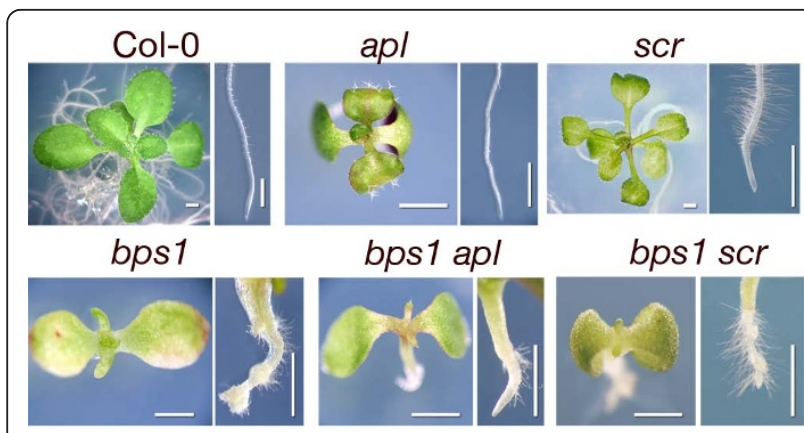

Figure 4 Neither phloem nor endodermal cell types are required for production or transmission of the bps 1 mobile compound. Top row: shoot and root phenotypes of 15-day Col-0 (left), the phloem-deficient apl (middle), and scarecrow (scr, right). Bottom row: shoot and root phenotypes of bps1 (left), the bps1 apl double mutant (center), and bps 1 scr double mutant (right). The double mutants show a leaf arrest phenotype that is similar to that of bps 1, indicating that the root-derived mobile compound is still synthesized and transmitted to the shoot. Seedlings shown here were grown at $22^{\circ} \mathrm{C}$ and photographed at 15 days. Size bars: $1 \mathrm{~mm}$. bps 1 and scr bps1 double mutants resembled the bps 1 single mutant (Figure 4, Table 3). Taken together, these data indicate that normal root development, including formation of the phloem and the endodermis, is not required for production and delivery of the bps 1 signal.

\section{Shoot responses to the bps 1 mobile root-derived compound}

The reversible arrest of shoot development in $b p s 1$ mutants correlates with a loss of auxin responses [9], but the underlying mechanism of arrest is unknown. To broaden our understanding of shoot responses in bps1, we carried out a series of time-course analyses where we analyzed leaf size, shape, the distribution of dividing cells, and stress responses (necrotic lesion formation and appearance of ROS).

Dividing cells were identified using the CYCB1;1::GUS reporter, a cell cycle reporter expressed in cells at the G2/M phase [34]. Patterns of CYCB1;1::GUS expression in Arabidopsis are well characterized; early leaf development shows a nearly uniform distribution of GUSstaining (i.e. dividing) cells, while later in development cell divisions become restricted to the leaf base and provascular tissue [35] (Figure 5). In bps1 mutants, the three-day leaf primordia largely matched wild type in terms of size, shape and $C Y C B 1 ; 1:: G U S$ expression patterns, however there were pronounced differences by day four. The four-day wild type leaf was much larger than that of bps1 and CYCB1;1::GUS-staining cells were distributed throughout, while the small four-day bps1-2 leaf had only a few CYCB1;1::GUS-staining cells. At day five, the wild-type leaf started to show distinct lamina expansion, and a slight tendency for there to be more GUSstaining cells toward its proximal end. By contrast, the five-day bps1 leaf showed no sign of lamina expansion, and few CYCB1;1::GUS-staining cells. The six-day wildtype leaf showed a strong reduction of $C Y C B 1 ; 1:$ GUSstaining cells at the distal end, and was much larger than the five-day wild-type leaf, while the corresponding bps 1

Table 3 shr and scr radial patterning defects do not suppress the bps 1 shoot phenotype

\begin{tabular}{cccccccc}
\hline & & \multicolumn{5}{c}{ Shoot Phenotypes Observed } & \\
\cline { 3 - 6 } $\begin{array}{c}\text { Plants } \\
\text { analyzed }\end{array}$ & $\begin{array}{c}\text { Total } \\
\text { (n) }\end{array}$ & $\begin{array}{c}\text { wild } \\
\text { type } \\
\text { (n) }\end{array}$ & $\begin{array}{c}\text { scr } \\
\text { (n) }\end{array}$ & $\begin{array}{c}\text { shr } \\
\text { (n) }\end{array}$ & $\begin{array}{c}\text { bps1 } \\
\text { (n) }\end{array}$ & $\begin{array}{c}\text { Double } \\
\text { mutant } \\
\text { (n) }\end{array}$ & $\chi^{2}$ \\
\hline $\begin{array}{c}\text { scr3-9 bps1-2 } \\
\text { F3 }\end{array}$ & 721 & $\mathrm{n} / \mathrm{a}$ & 545 & $\mathrm{n} / \mathrm{a}$ & $\mathrm{n} / \mathrm{a}$ & 176 & $0.134^{\mathrm{a}}$ \\
shr bps1-2 F2 & 1040 & 576 & $\mathrm{n} / \mathrm{a}$ & 194 & 270 & $\mathrm{n} / \mathrm{a}$ & $0.528^{\mathrm{b}}$ \\
shr & 167 & 129 & $\mathrm{n} / \mathrm{a}$ & 38 & $\mathrm{n} / \mathrm{a}$ & $\mathrm{n} / \mathrm{a}$ & $0.449^{\mathrm{a}}$ \\
bps1-2 & 191 & 146 & $\mathrm{n} / \mathrm{a}$ & $\mathrm{n} / \mathrm{a}$ & 45 & $\mathrm{n} / \mathrm{a}$ & $0.211^{\mathrm{a}}$ \\
\hline
\end{tabular}

$\mathrm{n} / \mathrm{a}$, not applicable

a Critcal $\chi^{2}$ values at $95 \%$ confidence is 3.841 when $d f=1$.

${ }^{\mathrm{b}}$ Critical $\chi^{2}$ values at $95 \%$ confidence is 5.991 when $d f=2$. 


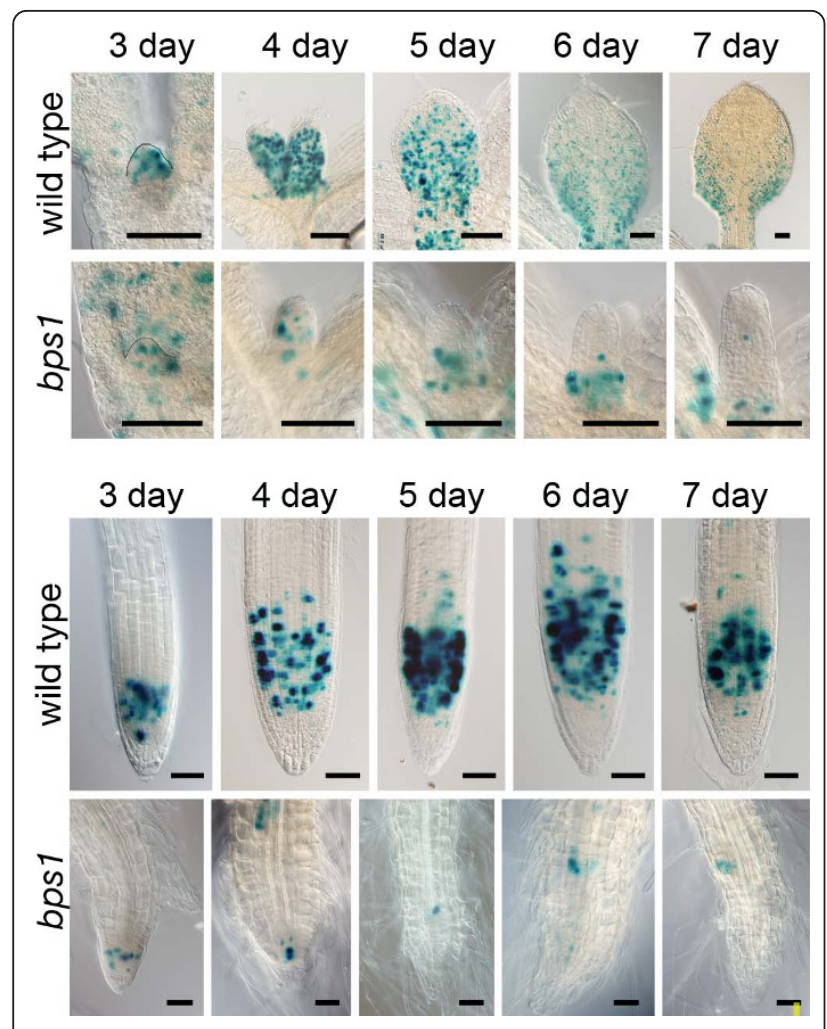

Figure 5 Growth and cell cycle progress are diminished in bps 1 leaves and roots. GUS stained tissue from bps 1 and wild-type plants carrying CYCB 1;1::GUS transgene. Top set: representative leaves, harvested at the same time daily (days three through seven). Bottom set: roots from the same time points. For both organs, severe effects on growth and development were obvious in bps 1 by day four, and included dramatic reduction in the number of G2/M phase cells. Size bar $=0.1 \mathrm{~mm}$ (leaves), and $0.05 \mathrm{~mm}$ (roots).

leaf was largely unchanged. By day seven, the wild-type leaf was even larger and the few $C Y C B 1 ; 1:: G U S$ expressing cells were at the leaf base. Similarly, the bps 1 sevenday leaf had only a few CYCB1;1::GUS-staining cells, and most were restricted to the leaf base. This analysis revealed a fully penetrant leaf arrest phenotype. In addition, despite the striking reduction in numbers of dividing cells, the apical/basal spatial control of cell divisions appeared to be intact.

While carrying out this analysis of leaf development, we also compared patterns of $C Y C B 1 ; 1:: G U S$-staining in roots (Figure 5). In the wild type, $C Y C B 1 ; 1:: G U S$-staining patterns were restricted to the root meristem, as has been described previously [36], and a similar pattern was observed between days three and seven. By contrast, at all time points, the bps1 root had fewer $C Y C B 1 ; 1:$ : GUS-staining cells.

The bps 1 mutant analysis revealed occasional necrotic lesions on bps 1 cotyledons (Figure 6A). To assess a possible relationship between these lesions and leaf arrest,
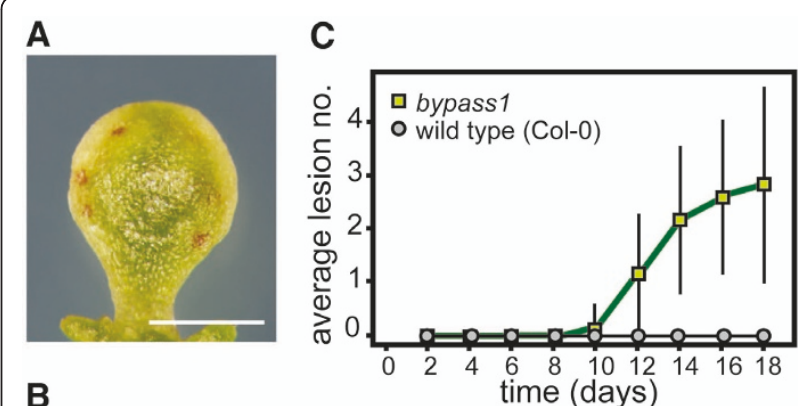

B time (days)

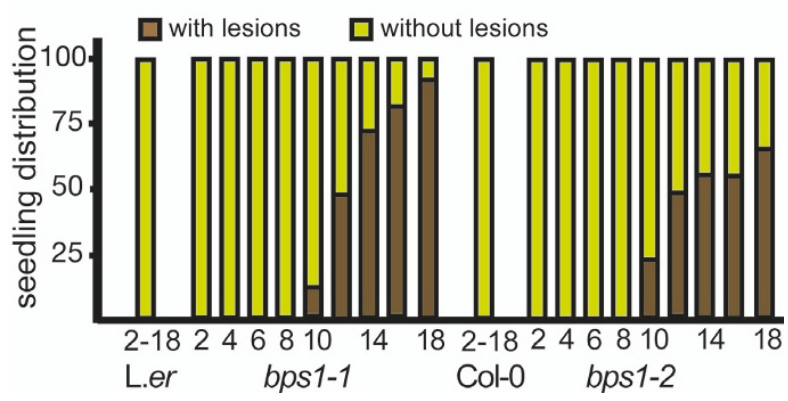

Figure 6 Necrotic Lesion formation is a late and not fully penetrant phenotype in bps 1 mutants. (A). 14-day bps 1-1

cotyledon with necrotic lesions. (B). Onset and penetrance of necrotic lesion formation. Green bars represent the percent of plants with no lesions, and brown bars represent the percent with one or more necrotic lesion. Neither the L.er nor Col-0 wild type produced any necrotic lesions, whereas for both bps 1-1 and bps 1-2 mutants formed necrotic lesion starting between day 8 and 10, after which lesion number increased steadily. $N=84$ (L.er), 140 (bps 1-1), 48 (Col-0), 80 (bps 1-2) (C) Average number of lesions per seedling. The number of lesions per seedling is depicted as a function of time, and bars show standard deviation. size bar $=1 \mathrm{~mm}$.

we carried out another time-course analysis, this time examining wild type and bps 1 mutants for necrotic lesion formation. In bps 1 mutants, necrotic lesions began to appear between 8 and 10 days. They were restricted to cotyledons, and never observed on leaves, hypocotyls, or roots, and necrotic lesions were never observed on the wild type (Col-0 or L.er) (Figure 6B). More bps1-1 seedlings formed necrotic lesions than bps1-2, and by day $18,92 \%$ of the bps1-1 plants had at least one necrotic lesion. The average lesion number per plant was highly variable, and increased over time (Figure 6C); by day 18 the bps1-2 mutants had between zero and seven necrotic lesions. Both bps1-1 and bps1-2 are null alleles [1], and so we attribute the difference in lesion formation to their genetic backgrounds (Col-0 for bps1-2 and L.er for bps1-1).

Because lesion formation is typically preceded by reactive oxygen species (ROS) [37-40], we compared ROS in bps 1 and wild type shoots using diaminobenzadine (DAB) to assay for hydrogen peroxide $\left(\mathrm{H}_{2} \mathrm{O}_{2}\right)$ and nitroblue tetrazolium (NBT) to assay for superoxide. Because the bps1-1 allele showed a more robust necrotic lesion 
phenotype, these analyses used bps1-1 and L. er. Both staining procedures produced a strong reaction in the vascular tissue, consistent with a role for ROS in lignification $[41,42]$. We found $\mathrm{H}_{2} \mathrm{O}_{2}$ in the 14-day bps 1 cotyledons, typically in positions surrounding the developing necrotic lesions (Figure 7A), but did not observe any nonvascular staining in the wild type cotyledon (data not shown). Additionally, we did not detect $\mathrm{H}_{2} \mathrm{O}_{2}$ in bps1 leaves. Similarly, superoxide was primarily associated with vascular tissue in wild type leaves, and it was nearly absent from the leaves of bps 1 mutants (Figure $7 \mathrm{~B})$. Because accumulation patterns of these two ROS were similar for the wild type and bps 1 mutants, severe oxidative stress does not appear to cause the bps1 leaf developmental arrest.

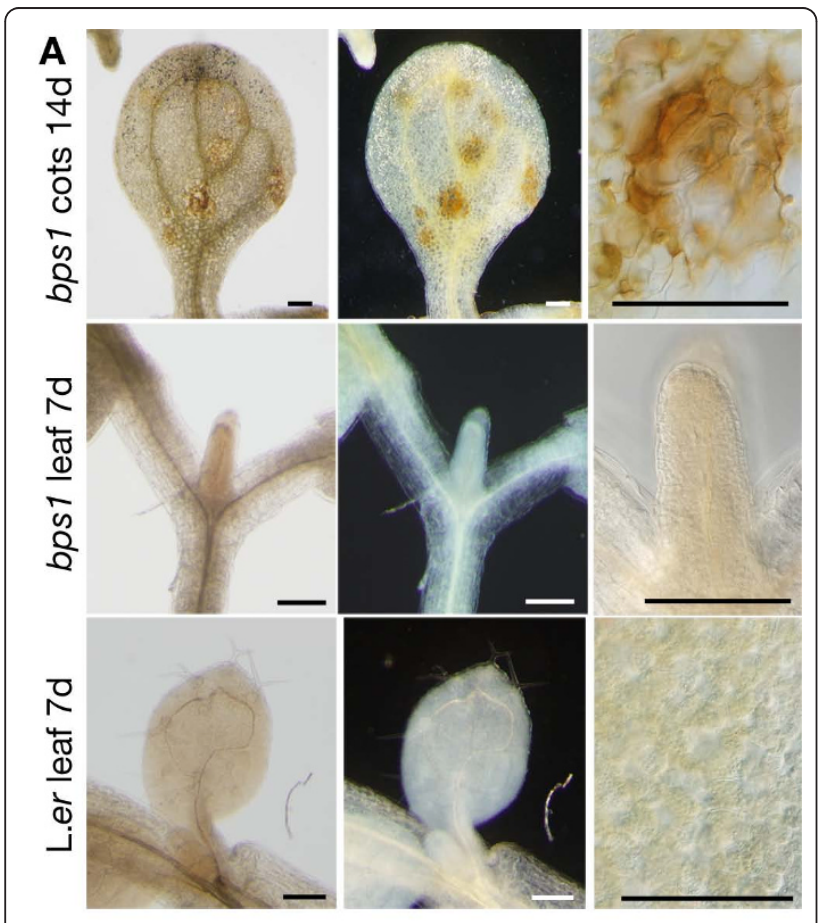

B

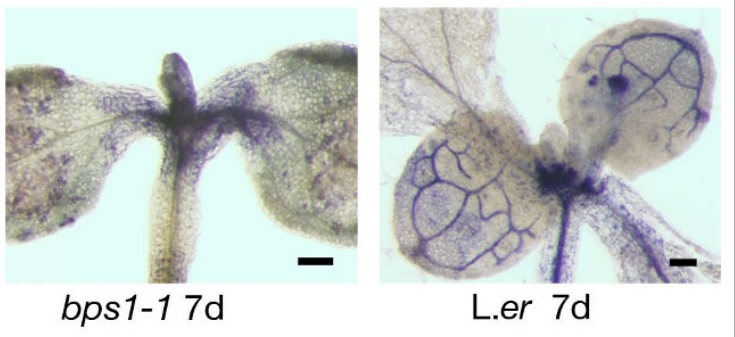

Figure 7 ROS is not increased in arrested bps 1 leaves. (A). Hydrogen peroxide, visualized using DAB, was found in necrotic lesions and associated with vascular tissue, but it was not elevated in the bps1 leaf. (B) Superoxide, visualized using NBT staining, was found associated with vascular tissue, but it was not elevated in the bps 1 leaf. Bars $=200 \mu \mathrm{m}$

\section{Discussion}

Physiological studies have implicated long distance signaling as a link between the development and physiology of roots and shoots [20]. However, only a small number of long-distance signaling pathways have been verified molecularly. In Arabidopsis bps 1 mutants, the non-cell-autonomous activity of mutant roots suggests that BPS1 might function to limit the synthesis of a root-derived mobile signaling molecule [9].

\section{Capacity to Synthesize the BPS1 Mobile Compound}

A central feature of bps1 mutants is that the root is the source of a biologically active mobile compound, which we refer to as the bps 1 signal. Here, we extended our understanding of the conditions under which the mutant root produces this compound. Previously we showed that cutting off the root led to rescue of the first leaf pair [9]. Indeed, we have now found that arresting post-embryonic bps 1 root growth also resulted in rescue of leaf development. However, in contrast to root excision, the first leaf pair was only mildly rescued, and strong rescue was delayed until leaf three. These observations indicate that the bps1 root, despite postembryonic arrest, retained a transient ability to supply the bps 1 signal to the shoot.

We used two related approaches to arrest postembryonic root growth: we caused arrest through the depletion of GSH either genetically (using the rml1-1 mutant) or chemically (using BSO). In both cases, the first leaf pair in GSH-depleted bps1 was larger than that of untreated bps 1 mutants, and the first leaf pair of bps 1 rml1-1 double mutants were consistently larger than that of BSO-grown bps1. Here, a larger leaf size probably reflects an earlier block to GSH synthesis in the mutant, and therefore an earlier reduction in bps1 signal synthesis.

Similarly, we found that restoring development of bps 1 roots (by GSH provision to bps 1 rml1-1 seedlings) reinstated arrest of leaf development. The extended capacity to produce and respond to the mobile compound is in line with physiological studies of drought-evoked long distance signaling, which has been documented in diverse plants, and at varying developmental stages [4].

A possibly less obvious question is why growtharrested roots (i.e. bps 1 rml1-1 double mutants and BSO-grown seedlings) show a decreased ability to arrest shoot growth. One possibility is that bps1 signal synthesis has a direct requirement for GSH. Alternatively, either synthesis or transmission to the shoot requires active root growth and cell division.

The maintenance of shoot arrest in apl bps 1 double mutants is consistent with a link to root growth. Although apl mutants have determinate roots [29], growth ceases later than for rml1-1 or BSO-treated 
plants, and the apl bps1 analysis was carried out prior to evidence of root cell division arrest. However, if root growth is a requirement for bps1 signal synthesis, then we would need to be able to explain constitutive synthesis of bps1 signal in bps1 mutants, which show primary root arrest soon after germination. One possibility is that synthesis is sustained by lateral roots, which initiate repeatedly. Alternatively, bps1 roots (including the primary) expand radially, and this radial growth might also sustain synthesis of the bps1 signal.

\section{bps1 signal transmission}

Movement of the bps1 signal from the root to the shoot is likely to use the plant's vascular system. Two vascular tissues are specialized for long-distance movement: the phloem, which transports photosynthate, and also mRNAs and proteins; and the xylem, which primarily transports water and dissolved nutrients. Here, we found that the shoot undergoes arrest in bps1 apl double mutants, which lack phloem [29]. The simplest conclusion is that the bps1 signal moves in the xylem. However, this conclusion is not definitive, because the very small size of bps1 apl double mutants doesn't preclude movement by diffusion.

\section{Shoot responses to the mobile bps 1 signal}

The small leaf size and reduced number of CYCB1;1:: GUS expressing cells are a fully penetrant bps 1 phenotype. Strikingly, although reduced in number, the pattern of CYCB1;1::GUS-expressing cells mimicked the wild type pattern: leaf primordia showed an even distribution of diving cells, but as the mutant leaves matured, dividing cells were restricted to the base of the leaf. The retention of a normal pattern of dividing cells shows that some aspects of leaf developmental programming persist in bps1 mutants. This result hints that instead of altering development, the bps1 signal might instead disrupt the link between development and cell cycle control.

Another phenotype in bps1 mutants is the formation of necrotic lesions. These were late-appearing and not fully penetrant. Necrotic lesions have been observed in a wide range of Arabidopsis mutants. These include plants with defects in syntaxin genes [43], and mutants with defects in the cytochrome P450 gene CYP83B1, which results in excess auxin synthesis [44]. Necrosis is typically associated with plant defense responses, and can be a secondary consequence of elevated expression of defense genes, such as observed in the developmental mutant asymmetric leaf 1 [45] and in response to phosphate deficiency [46,47].

\section{Conclusions}

The results presented here support the phenomenon of shoot arrest by a root-derived molecule in bps 1 mutants. A key question raised by discovery and characterization of this mutant is whether the bps1 mutation exposes a novel root-to-shoot signaling molecule or a metabolic intermediate with toxic effects on shoot development. The crucial difference between these two concepts is that a novel root-to-shoot signaling molecule would be present in the wild type, while a metabolic intermediate would only accumulate in bps 1 mutants. Because the synthesis of the root-derived molecule requires postembryonic root development and aerial organs appear to arrest growth prior to showing any signs of toxicity (necrosis), we tend to favor the hypothesis that bps 1 reveals a novel root-to-shoot signaling pathway. A full resolution of this issue awaits biochemical identification of this mobile molecule. Regardless of the nature of the root-derived compound, it should be pointed out that under either scenario the bps1 mutation has unveiled a molecule with potent biological activity. Despite the impact of root-to-shoot communication on plant productivity, the molecular mechanisms involved are poorly understood. The bps 1 mutation could be utilized as a tool to begin to dig into the pathways that both synthesize and respond to root-derived growth modulators.

\section{Methods \\ Plant Growth}

All seeds were cold-shocked for 2-4 days in darkness at $4^{\circ} \mathrm{C}$, and most grown in 24 hour light at the $22^{\circ} \mathrm{C}$, unless noted otherwise. Growth media composition is $0.5 \mathrm{X}$ MS salts (Caisson labs), 1\% sucrose, 0.5g/l MES, pH 5.8, $0.8 \%$ phytoblend agar (Caisson Laboratories). Seedlings were grown in Conviron TC30 growth chambers under light and temperature regimes as described.

\section{Plant Materials}

Mutant alleles used: bps1-2 (Col), bps1-1 (L.er), rml1-1 (Col, received from Z.R. Sung), scr-3 (Col, CS3997), shr (Col, SALK_002744), apl (Col, received from M. Bonke), and CYCB1;1::GUS seeds were received from J.L. Celenza.

\section{GUS Staining}

The CYCB1;1::GUS transgene was crossed into both bps1-1 and bps1-2, and F3 lines homozygous for the transgene and segregating for bps1 were identified. We plated these lines (and control wild-type transgenic) on normal growth media, and subjected them to a 2-7 day cold shock $\left(4^{\circ} \mathrm{C}\right)$. Each day, plates were transferred to a $22^{\circ} \mathrm{C}$ growth chamber. GUS staining followed previously published protocols [48].

\section{Conditional Root Arrest}

Arrest of roots using BSO was carried out by making our standard GM (above), and supplementing it to $2.5 \mathrm{mM}$ BSO (DL-Buthionine-[S,R]-sulfoximine, Sigma), 
and bps1-2 rml1-1 double mutants were generated by standard methods. For both BSO and rmll experiments, plants were grown in short day ( 8 hours light/16 hours dark) at $22^{\circ} \mathrm{C}$. To reinstate root growth of rml1-1, we supplemented the media to $750 \mu \mathrm{M}$ glutathione (Acros Organics) [27]. To test whether the number of plants producing broad leaves upon root excision in the presence of GSH was statistically different from that observed in the absence of GSH (Table 2), we performed hypothesis testing for proportions using the $\mathrm{Z}$-score method. If GSH were the root-shoot signal, we would predict that the number of plants forming broad leaves under GSH+ conditions would be less than under GSH-conditions (the null hypothesis). The statistical tests indicate that the number of plants producing leaves in the presence of GSH is not less than or equal to the number producing leaves in the absence of GSH. Because the calculated P value is low, we must reject the null hypothesis in support of the alternative hypothesis that the number of plants producing leaves under GSH+ conditions is greater than GSHconditions. This indicated that the root-to-shoot signal is not GSH.

\section{Stress symptom analyses}

Necrotic lesion formation was assessed by a visual inspection of bps1 and wild type seedlings. All organs of the investigated seedlings were examined on alternate days. To visualize patterns of $\mathrm{H}_{2} \mathrm{O}_{2}$ in seedlings (wild type and bps1), we used 3,3'-diaminobenzidine (DAB) staining as described $[49,50]$. We infiltrated $0.1 \%(\mathrm{~W} / \mathrm{V})$ DAB (Sigma), pH3.8, and allowed staining to progress for 4-6 hours. After staining, samples were cleared in $70 \%$ ethanol, and then transferred to $40 \% \mathrm{w} / \mathrm{v}$ glycerol, mounted on glass slides, and examined on Olympus BX50 and Olympus SZX16 microscopes. Visualization of superoxide patterns used nitroblue tetrazolium staining protocols as described [51,52].

\section{Acknowledgements \\ We would like to thank Dong-Keun Lee and Emma Adhikari for useful discussions of the work and for proofreading. This project supported by the National Research Initiative competitive grant no. 2008-35304-04488 (to LES) from the USDA National Institute of Food and Agriculture, by award IOB- 0922288 (to LES) from the National Science Foundation, and an award from NSF-supported BioURP award to $\mathrm{CM}$ and $\mathrm{NIH}$ training grant support to JMVN (NIH training grant number 5 T32 GM007464).}

\section{Author details}

'Department of Biology, University of Utah, 257 South 1400 East, Salt Lake City, Utah, 84112, USA. 'Biology Department, Duke University, Durham, North Carolina, 27708, USA.

\section{Authors' contributions}

JMVN carried out rm/1 and BSO root arrest experiments and the apl bps1, shr bps1, and scr bps 1 double mutant analyses. CM carried out analyses of ROS, characterized the lesion formation phenotype, and analyzed shoot phenotypes following resumption of root development. LES carried out the
pCYCB1;1::GUS analyses. LES and JMVN planned the project together, and the manuscript was primarily written by LES with assistance from JMVN. All authors read and approved the final manuscript.

Received: 14 May 2010 Accepted: 3 February 2011

Published: 3 February 2011

\section{References}

1. Last RL, Jones AD, Shachar-Hill Y: Towards the plant metabolome and beyond. Nat Rev Mol Cell Biol 2007, 8:167-174.

2. Sawada Y, Akiyama K, Sakata A, Kuwahara A, Otsuki H, Sakurai T, Saito K, Hirai MY: Widely targeted metabolomics based on large-scale MS/MS data for elucidating metabolite accumulation patterns in plants. Plant Cell Physiol 2009, 50:37-47.

3. Urano K, Kurihara Y, Seki M, Shinozaki K: 'Omics' analyses of regulatory networks in plant abiotic stress responses. Curr Opin Plant Biol 2010, 13:132-138.

4. Giavalisco P, Hummel J, Lisec J, Inostroza AC, Catchpole G, Willmitzer L: High-resolution direct infusion-based mass spectrometry in combination with whole $13 \mathrm{C}$ metabolome isotope labeling allows unambiguous assignment of chemical sum formulas. Anal Chem 2008, 80:9417-9425.

5. Cui $Q$, Lewis IA, Hegeman AD, Anderson ME, Li J, Schulte CF, Westler WM, Eghbalnia HR, Sussman MR, Markley JL: Metabolite identification via the Madison Metabolomics Consortium Database. Nat Biotechnol 2008, 26:162-164.

6. Meyer RC, Steinfath M, Lisec J, Becher M, Witucka-Wall H, Torjek O, Fiehn O, Eckardt A, Willmitzer L, Selbig J, Altmann T: The metabolic signature related to high plant growth rate in Arabidopsis thaliana. Proc Natl Acad Sci USA 2007, 104:4759-4764.

7. Lisec J, Meyer RC, Steinfath M, Redestig H, Becher M, Witucka-Wall H, Fiehn O, Torjek O, Selbig J, Altmann T, Willmitzer L: Identification of metabolic and biomass QTL in Arabidopsis thaliana in a parallel analysis of RIL and IL populations. Plant J 2008, 53:960-972.

8. Hannah MA, Caldana C, Steinhauser D, Balbo I, Fernie AR, Willmitzer L: Combined transcript and metabolite profiling of Arabidopsis grown under widely variant growth conditions facilitates the identification of novel metabolite-mediated regulation of gene expression. Plant Physiol 2010, 152:2120-2129.

9. Van Norman JM, Frederick RL, Sieburth LE: BYPASS1 negatively regulates a root-derived signal that controls plant architecture. Curr Biol 2004, 14:1739-1746.

10. Van Norman JM, Sieburth LE: Dissecting the biosynthetic pathway for the bypass1 root-derived signal. Plant J 2007, 49:619-628.

11. Kang YW, Kim RN, Cho HS, Kim WT, Choi D, Pai HS: Silencing of a BYPASS1 homolog results in root-independent pleiotrophic developmental defects in Nicotiana benthamiana. Plant Mol Biol 2008, 68:423-437.

12. Booker J, Sieberer T, Wright W, Williamson L, Willett B, Stirnberg P, Turnbull C, Srinivasan M, Goddard P, Leyser O: MAX1 encodes a cytochrome P450 family member that acts downstream of MAX3/4 to produce a carotenoid-derived branch-inhibiting hormone. Dev Cell 2005, 8:443-449.

13. Zou J, Zhang S, Zhang W, Li G, Chen Z, Zhai W, Zhao X, Pan X, Xie Q, Zhu L: The rice HIGH-TILLERING DWARF1 encoding an ortholog of Arabidopsis MAX3 is required for negative regulation of the outgrowth of axillary buds. Plant J 2006, 48:687-698.

14. Sorefan K, Booker J, Haurogne K, Goussot M, Bainbridge K, Foo E, Chatfield S, Ward S, Beveridge C, Rameau C, Leyser O: MAX4 and RMS1 are orthologous dioxygenase-like genes that regulate shoot branching in Arabidopsis and pea. Genes Dev 2003, 17:1469-1474.

15. Morris SE, Turnbull CG, Murfet IC, Beveridge CA: Mutational analysis of branching in pea. Evidence that Rms1 and Rms5 regulate the same novel signal. Plant Physiol 2001, 126:1205-1213.

16. Stirnberg $P$, van De Sande $K$, Leyser HM: MAX1 and MAX2 control shoot lateral branching in Arabidopsis. Development 2002, 129:1131-1141.

17. Bennett T, Sieberer T, Willett B, Booker J, Luschnig C, Leyser O: The Arabidopsis MAX pathway controls shoot branching by regulating auxin transport. Curr Biol 2006, 16:553-563.

18. Gomez-Roldan V, Fermas S, Brewer PB, Puech-Pages V, Dun EA, Pillot JP, Letisse F, Matusova R, Danoun S, Portais JC, Bouwmeester H, Bécard G, Beveridge CA, Rameau C, Rochange SF: Strigolactone inhibition of shoot branching. Nature 2008, 455:189-194. 
19. Umehara M, Hanada A, Yoshida S, Akiyama K, Arite T, Takeda-Kamiya N, Magome H, Kamiya Y, Shirasu K, Yoneyama K, Kyozuka J, Yamaguchi S: Inhibition of shoot branching by new terpenoid plant hormones. Nature 2008, 455:195-200

20. Davies W, Zhang J: Root Signals and the Regulation of Growht and Development of Plants in Drying Soil. Annual Rev Plant Physiol Plant mol Biol 1991, 42:55-76.

21. Mulholland BJ, Black CR, Taylor IB, Roberts JA, Lenton JR: Effect of soil compaction on barley (Hordeum vulgare L.) growth 1. Possible role for $\mathrm{ABA}$ as a root-sourced chemical signal. Journal of Experimental Botany 1996, 47:539-549.

22. Fujii H, Chiou TJ, Lin SI, Aung K, Zhu JK: A miRNA involved in phosphatestarvation response in Arabidopsis. Curr Biol 2005, 15:2038-2043.

23. Bari R, Datt Pant B, Stitt M, Scheible WR: PHO2, microRNA399, and PHR1 define a phosphate-signaling pathway in plants. Plant Physiol 2006, 141:988-999.

24. Forde BG: The role of long-distance signalling in plant responses to nitrate and other nutrients. J Exp Bot 2002, 53:39-43.

25. Tong H, Leasure CD, Hou X, Yuen G, Briggs W, He ZH: Role of root UV-B sensing in Arabidopsis early seedling development. Proc Natl Acad Sci USA 2008, 105:21039-21044.

26. Mikkelsen MD, Naur $\mathrm{P}$, Halkier BA: Arabidopsis mutants in the C-S lyase of glucosinolate biosynthesis establish a critical role for indole-3acetaldoxime in auxin homeostasis. Plant J 2004, 37:770-777.

27. Vernoux T, Wilson RC, Seeley KA, Reichheld JP, Muroy S, Brown S, Maughan SC, Cobbett CS, Van Montagu M, Inze D, May MJ, Sung ZR: The ROOT MERISTEMLESS1/CADMIUM SENSITIVE2 gene defines a glutathione-dependent pathway involved in initiation and maintenance of cell division during postembryonic root development. Plant Cell 2000, $12: 97-110$

28. Cheng JC, Seeley KA, Sung ZR: RML1 and RML2, Arabidopsis genes required for cell proliferation at the root tip. Plant Physiol 1995, 107:365-376

29. Bonke M, Thitamadee S, Mahonen AP, Hauser MT, Helariutta Y: APL regulates vascular tissue identity in Arabidopsis. Nature 2003, 426:181-186.

30. Benfey PN, Linstead PJ, Roberts K, Schiefelbein JW, Hauser MT, Aeschbacher RA: Root development in Arabidopsis: four mutants with dramatically altered root morphogenesis. Development 1993, 121:53-62.

31. Scheres B, DiLaurenzio L, Willemsen V, Hauser MT, Janmaat $K$, Weisbeek $P$, Benfey PN: Mutations affecting the radial organization of the Arabidopsis root display specific defects throughout the radial axis. Development 1995, 121:53-62

32. Di Laurenzio L, Wysocka-Diller J, Malamy JE, Pysh L, Helariutta Y, Freshour G, Hahn MG, Feldmann KA, Benfey PN: The SCARECROW gene regulates an asymmetric cell division that is essential for generating the radial organization of the Arabidopsis root. Cell 1996, 86:423-433.

33. Helariutta Y, Fukaki H, Wysocka-Diller J, Nakajima K, Jung J, Sena G, Hauser MT, Benfey PN: The SHORT-ROOT gene controls radial patterning of the Arabidopsis root through radial signaling. Cell 2000, 101:555-567.

34. Colon-Carmona A, You R, Haimovitch-Gal T, Doerner P: Technical advance: spatio-temporal analysis of mitotic activity with a labile cyclin-GUS fusion protein. Plant J 1999, 20:503-508.

35. Kang J, Dengler N: Cell cycling frequency and expression of the homeobox gene ATHB-8 during leaf vein development in Arabidopsis. Planta 2002, 216:212-219.

36. Beeckman T, Burssens $S$, Inze D: The peri-cell-cycle in Arabidopsis. J Exp Bot 2001, 52:403-411

37. Dong X, Mindrinos M, Davis KR, Ausubel FM: Induction of Arabidopsis defense genes by virulent and avirulent Pseudomonas syringae strains and by a cloned avirulence gene. Plant Cell 1991, 3:61-72

38. Heath MC: Hypersensitive response-related death. Plant Mol Biol 2000 44:321-334.

39. Kovtun Y, Chiu WL, Tena G, Sheen J: Functional analysis of oxidative stress-activated mitogen-activated protein kinase cascade in plants. Proc Natl Acad Sci USA 2000, 97:2940-2945

40. Manzano D, Fernandez-Busquets X, Schaller H, Gonzalez V, Boronat A, Arro M, Ferrer $A$ : The metabolic imbalance underlying lesion formation in Arabidopsis thaliana overexpressing farnesyl diphosphate synthase (isoform 1S) leads to oxidative stress and is triggered by the developmental decline of endogenous HMGR activity. Planta 2004, 219:982-992

41. Ogawa K, Kanematsu S, Asada K: Generation of superoxide anion and localization of CuZn-superoxide dismutase in the vascular tissue of spinach hypocotyls: their association with lignification. Plant Cell Physiol 1997, 38:1118-1126.

42. Whetten RW, MacKay JJ, Sederoff RR: Recent Advances in Understanding Lignin Biosynthesis. Annu Rev Plant Physiol Plant Mol Biol 1998, 49:585-609.

43. Zhang Z, Lenk A, Andersson MX, Gjetting T, Pedersen C, Nielsen ME, Newman MA, Hou BH, Somerville SC, Thordal-Christensen H: A lesionmimic syntaxin double mutant in Arabidopsis reveals novel complexity of pathogen defense signaling. Mol Plant 2008, 1:510-527.

44. Smolen G, Bender J: Arabidopsis cytochrome P450 cyp83B1 mutations activate the tryptophan biosynthetic pathway. Genetics 2002, 160:323-332.

45. Nurmberg PL, Knox KA, Yun BW, Morris PC, Shafiei R, Hudson A, Loake GJ: The developmental selector AS1 is an evolutionarily conserved regulator of the plant immune response. Proc Natl Acad Sci USA 2007, 104:18795-18800.

46. Wu P, Ma L, Hou X, Wang M, Wu Y, Liu F, Deng XW: Phosphate starvation triggers distinct alterations of genome expression in Arabidopsis roots and leaves. Plant Physiol 2003, 132:1260-1271.

47. Hammond JP, Bennett MJ, Bowen HC, Broadley MR, Eastwood DC, May ST, Rahn C, Swarup R, Woolaway KE, White PJ: Changes in gene expression in Arabidopsis shoots during phosphate starvation and the potential for developing smart plants. Plant Physiol 2003, 132:578-596.

48. Deyholos MK, Cavaness GF, Hall B, King E, Punwani J, Van Norman J, Sieburth LE: VARICOSE, a WD-domain protein, is required for leaf blade development. Development 2003, 130:6577-6588.

49. Thordal-Christensen H, Zhang Z, Wei Y, Collinge DB: Subcellular localization of $\mathrm{H} 2 \mathrm{O} 2$ in plants. $\mathrm{H} 2 \mathrm{O} 2$ accumulation in papillae and hypersensitive response during the barley-powdery mildew interaction. Plant Journal 1997, 11:1187-1194.

50. Ahn IP, Kim S, Lee YH, Suh SC: Vitamin B1-induced priming is dependent on hydrogen peroxide and the NPR1 gene in Arabidopsis. Plant Physiol 2007, 143:838-848

51. Hoffmann A, Hammes E, Plieth C, Desel C, Sattelmacher B, Hansen U-P. Effect of $\mathrm{CO} 2$ supply on formation of reactive oxygen species in Arabidopsis thaliana. Protoplasma 2005, 227:3-9.

52. Giraud E, Ho LH, Clifton R, Carroll A, Estavillo G, Tan YF, Howell KA, Ivanova A, Pogson BJ, Millar AH, Whelan J: The absence of ALTERNATIVE OXIDASE1a in Arabidopsis results in acute sensitivity to combined light and drought stress. Plant Physiol 2008, 147:595-610.

doi:10.1186/1471-2229-11-28

Cite this article as: Van Norman et al:: BYPASS1: synthesis of the mobile root-derived signal requires active root growth and arrests early leaf development. BMC Plant Biology 2011 11:28.

\section{Submit your next manuscript to BioMed Central and take full advantage of:}

- Convenient online submission

- Thorough peer review

- No space constraints or color figure charges

- Immediate publication on acceptance

- Inclusion in PubMed, CAS, Scopus and Google Scholar

- Research which is freely available for redistribution

Submit your manuscript at www.biomedcentral.com/submit
C Biomed Central 